\title{
Contactless Inter-Tier Communication for Heterogeneous 3-D ICs
}

DOI:

10.1109/ISCAS.2017.8050946

\section{Document Version}

Accepted author manuscript

Link to publication record in Manchester Research Explorer

\section{Citation for published version (APA):}

Pavlidis, V., \& Papistas, I. (Accepted/In press). Contactless Inter-Tier Communication for Heterogeneous 3-D ICs. In International Symposium on Circuits and Systems https://doi.org/10.1109/ISCAS.2017.8050946

\section{Published in:}

International Symposium on Circuits and Systems

\section{Citing this paper}

Please note that where the full-text provided on Manchester Research Explorer is the Author Accepted Manuscript or Proof version this may differ from the final Published version. If citing, it is advised that you check and use the publisher's definitive version.

\section{General rights}

Copyright and moral rights for the publications made accessible in the Research Explorer are retained by the authors and/or other copyright owners and it is a condition of accessing publications that users recognise and abide by the legal requirements associated with these rights.

\section{Takedown policy}

If you believe that this document breaches copyright please refer to the University of Manchester's Takedown Procedures [http://man.ac.uk/04Y6Bo] or contact uml.scholarlycommunications@manchester.ac.uk providing relevant details, so we can investigate your claim.

\section{OPEN ACCESS}




\title{
Contactless Inter-Tier Communication for Heterogeneous 3-D ICs
}

\author{
Ioannis A. Papistas and Vasilis F. Pavlidis \\ Advanced Processor Technologies Group \\ School of Computer Science, The University of Manchester \\ \{papistai, pavlidis\}@cs.man.ac.uk
}

\begin{abstract}
A heterogeneous contactless transceiver circuit is designed to provide half duplex communication for a 3-D system considering specific bonding constraints. The system is composed of two tiers and is integrated face-to-back to support fluidic sensing. Communication between the tiers is achieved through inductive links. Each tier is considered to be fabricated in a different technology node to enable low manufacturing cost and benefit from the advantages each technology offers. Both the uplink and downlink transceivers achieve data rates that reach 1 Gbps with non-return-to-zero data encoding. Energy efficiency is the primary objective, with the uplink dissipating $4.93 \mathrm{~mW}$ and the downlink $10.53 \mathrm{~mW}$. A $5.2 \times$ power reduction is achieved when using heterogeneous technologies, compared to a stateof-the-art $0.35 \mu \mathrm{m}$ transceiver, while the dissipated energy is decreased by $34 \%$ as compared to a state-of-the-art $65 \mathrm{~nm}$ transceiver.
\end{abstract}

\section{INTRODUCTION}

Heterogeneous 3-D integration provides a promising platform for edge devices of the Internet-of-Things (IoT) [1] comprising multi-functional, small form factor, and low power systems [2]. A heterogeneous 3-D system is composed of stacked integrated circuits, manufactured in disparate technologies. Digital, analog, and sensors circuits can thus coexist in a single 3-D package reducing the form factor of the system [3]. This approach is suitable for Lab-on-Chip (LoC) [4] or the IoT applications where multi-functionality and power are important.

Nevertheless, several challenges exist for developing heterogeneous 3-D systems, such as heat dissipation, testing, and inter-tier communication [3]. Considering inter-tier interconnects, through silicon vias (TSVs) provide high density signaling with low latency and power [5]. However, TSVs are an expensive means due to the related manufacturing complexity and possibly low yield [6]. Alternatively, contactless solutions based on ac coupling have been proposed [7]-[10].

Both inductive and capacitive contactless communication are compatible with conventional CMOS process and therefore no additional manufacturing steps are required for underpinning inter-tier communication [8], [11], [12]. Wireless interconnects offer several advantages to heterogeneous 3$\mathrm{D}$ integration including die detachability [8] and inter-tier communication without the requirement of level shifters [13].

This work was supported in part by EPSRC UK under Grant EP/M009238/1 and scholarships from the School of Computer Science and the Doctoral Training Centre at the University of Manchester.
However, capacitive coupling is limited to face-to-face bonding, supporting primarily two tier systems and therefore significantly narrowing the candidate applications for this communication mechanism. For example, face-to-face integration is not a feasible option for LoC applications where the sensing tier should be integrated face up. Due to these limitations, inductive links are investigated in this work.

Systems with functional heterogeneity have recently been explored, [14], where inductive links within a 3-D networkon-chip interconnect a multicore processor with accelerators on several tiers. Alternatively, an interface for memory control manufactured in a $65 \mathrm{~nm}$ technology is proposed in [15] where the channel of the transistors in the memory modules is elongated to emulate a $100 \mathrm{~nm}$ node. However, this circuit is inherently homogeneous, utilising only one technology node. In [16], despite the one technology node difference between the tiers, only digital systems are interconnected. Additionally, these systems target memory-processor communication where speed is of high importance. However, the nature of sensing applications requires a different design approach since power and cost are often more important than speed [17]. Thus, sensing circuits are usually implemented in older and therefore low cost technologies. Consequently, there is a significant technology gap between the sensor circuits and the digital circuits that control the sensor and process the sensory data.

In this paper, a transceiver circuit is proposed for a 3D system characterised by both functional and technological heterogeneity. The target system is a two tier system consisting of a processing tier and a sensing tier implemented, respectively, in a $65 \mathrm{~nm}$ [18] and a $0.35 \mu \mathrm{m}$ technology [19]. Homogeneous inductive links have been demonstrated in both of these technologies, where the most advanced manufacturing process for a prototype inductive link is a $65 \mathrm{~nm}$ technology [18]. The choice of the $0.35 \mu \mathrm{m}$ technology is due to the typical use of this technology for sensing circuits [19], [20]. In addition, these homogeneous links serve as a baseline for comparison.

The remainder of this paper is organised as follows. In Section II, the salient features of the envisioned two tier system are introduced. The design of the inductive link transceiver that provides communication between the tiers is presented in Section III, where related design tradeoffs are also explored. Finally, some conclusions are summarised in Section V. 


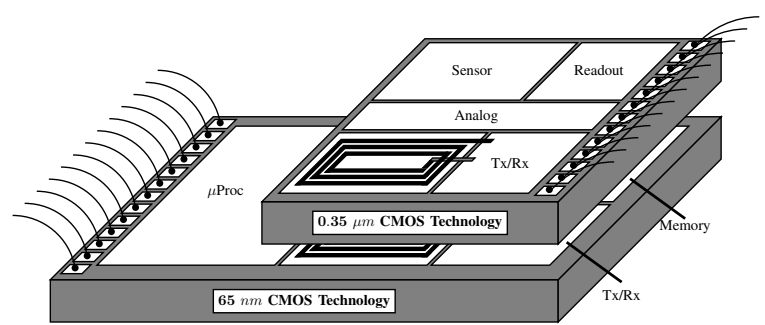

(a)



(b)

Fig. 1. (a) A heterogeneous 3-D stack including, respectively, one processing and one sensing tier and (b) coupling between the inductors depending upon the outer diameter of the inductors and communication distance.

\section{Heterogeneous Contactless 3-D System}

An overview of the heterogeneous 3-D system is presented in this section. Each tier is assumed to be manufactured at a different technology for improved yield and therefore lower cost. There exist several ways to integrate the two tiers of the system, which communicate wirelessly, including a 3-D $\mathrm{SiP}$ with contactless links as shown in Fig. 1(a), a hybrid 3-D stack with TSVs and contactless links, and a purely contactless system. A wireless approach is preferred over TSVs not to increase cost, where similar communication distances are achieved. Moreover, there is no need for level conversion, even though the supply voltage of each technology is different. Offchip communication between the tiers via wire bonds is also avoided to reduce the effect of parasitic impedance and achieve a higher performance and lower power as compared to an only wire bonded SiP System.

In this paper, emphasis is placed on the contactless intertier communication and the issues relating to the dissimilar manufacturing technologies of the tiers. Consequently, tradeoffs related to the design of the link including the coupling between the inductors, the area and power consumed by the inductors are explored.

The bottom tier of the system controls the sensor module and post processes the received data. The sensor tier is partitioned to the blocks that sense, transmit the sensed data, and receive the control data. Throughout this paper, uplink is the transceiver circuit with the transmitter module designed in the $65 \mathrm{~nm}$ technology and the receiver circuit in the $0.35 \mu \mathrm{m}$ technology, respectively. The uplink communicates the control data to the sensor tier. Conversely, for the transceiver of the downlink, the technologies for the transmitter and receiver circuits are $0.35 \mu \mathrm{m}$ and $65 \mathrm{~nm}$, respectively. The downlink transmits the sensed data to the processor tier.

The efficacy of the communication between the coupled inductors depends upon the achieved coupling given the separation distance $X$ and the outer diameter $d_{\text {out }}$ of the onchip inductors, as depicted in Fig. 1(b). Thinning the substrate of the upper tier improves the coupling coefficient, without excessively increasing the size of the on-chip inductor. For the chosen technologies, a minimum communication distance of $80 \mu \mathrm{m}$ is feasible without a significant cost overhead [19].

A minimum coupling of 0.1 is required to support inter-tier communication. Below 0.1, multiple amplification stages are required to receive the transmitted signal [13], which drasti- cally increase the power of the transceiver. As the objective is to maintain low power, these techniques are rather unsuitable and are therefore not considered.

The total power consumed by the inductive link is the sum of the power dissipated by both the uplink and downlink transceiver circuits. The goal is to minimise the power of both links, given the communication distance and outer diameter of the inductor pair. Since the two tiers are fabricated in different technologies, the power consumed by each tier is significantly affected by the supply voltage. The nominal supply voltage is respectively $V_{d d}=1.2 \mathrm{~V}$ and $V_{d d}=3.3 \mathrm{~V}$ for the $65 \mathrm{~nm}$ and $0.35 \mu \mathrm{m}$ technology. Given these voltages, greater power savings for the inductive link result by reducing the current drawn by the transceiver circuits of the sensor tier. Alternatively, the processing tier can be designed to satisfy the bandwidth requirements.

\section{Half Duplex Transceiver Design}

The proposed transceiver circuits for inter-tier communication are presented in this Section. In subsection III-A, the chosen coupled inductors are presented. In subsection III-B, the design of the proposed transceivers is described for both the uplink and downlink communication.

\section{A. Coupled Inductors and Signalling}

The design of the coupled inductors is presented in this subsection. An $R L C$ model of the inductors is described along with the utilised signalling approach.

The coupling coefficient for a communication distance of $X=80 \mu \mathrm{m}$ and increasing outer diameter $d_{\text {out }}$ is shown in Fig. 1(b). Ansys Maxwell [21] is used to accurately evaluate the coupling level. To achieve the minimum coupling an inductor with an outer diameter of $d_{\text {out }}=150 \mu \mathrm{m}$ is required. However, since the focus is on reducing the power dissipation, a larger inductor can be employed to increase the coupling level and facilitate the inter-tier communication. A coupling of $k=0.22$ is achieved using inductors with four turns, an outer diameter of $d_{\text {out }}=300 \mu \mathrm{m}$, and a wire width of $w=5 \mu \mathrm{m}$. To further increase the coupling, $d_{\text {out }}$ increases exponentially resulting in excessive area consumption. Additionally, to reduce the complexity and therefore power demand of the receiver circuit non-return-to-zero encoding is used for transmitting data.

A schematic of the coupled inductors is depicted in Fig. 2, where the corresponding $R L C$ characteristics are also shown. 


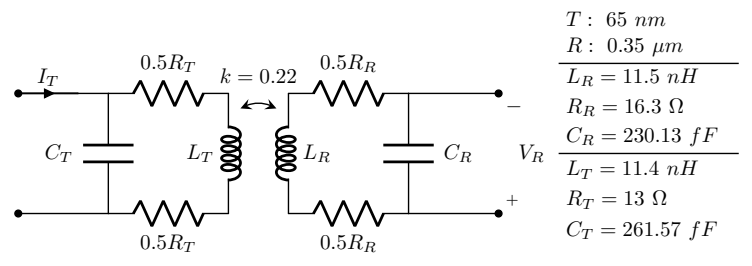

Fig. 2. The $R L C$ model of the inductive link pair.

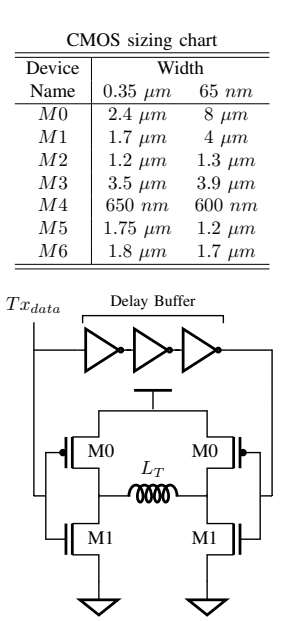

(a)

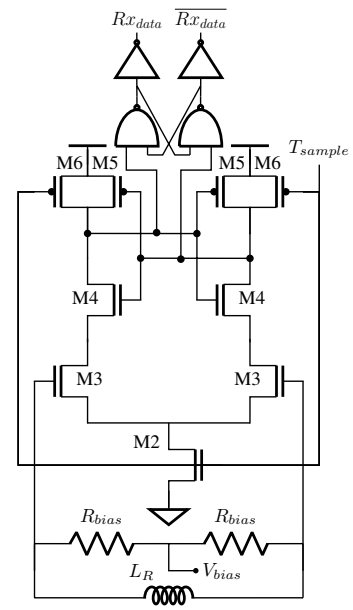

(b)
Fig. 3. The proposed transceiver circuit used for both the uplink and downlink communication, where (a) is the H-Bridge transmitter [23] and (b) is the synchronous sense amplifier receiver [23].

Despite the 4-node difference between the technologies of the tiers, the top interconnect layers used for the inductors exhibit similar physical characteristics (e.g. thickness). The illustrated $R L C$ characteristics for the paired inductors and the coupling coefficient $k$ are extracted from Ansys Maxwell [21] simulations and verified for manufacturability with Helic VeloceRF [22].

\section{B. Transceiver Design}

The proposed transceiver design for the communication between the processing and sensing tiers is described in this subsection. Commercial libraries are used for both the $65 \mathrm{~nm}$ technology [18] and the $0.35 \mu \mathrm{m}$ technology [19]. The nominal transistor length of each technology is utilised.

The transmitter consists of an $\mathrm{H}$-Bridge circuit driving the inductor, as shown in Fig. 3(a) [23]. For a specific physical distance between the tiers $X$, and coupling level $k$, the size of the transmitter and the sensitivity of the receiver circuit, i.e. the minimum amplitude of $V_{R x}$ that can be successfully sensed, are interdependent. In addition, the $M 0$ and $M 1$ devices shown in Fig. 3(a) must be appropriately sized to avoid any mismatch of the voltage that differentially drives the inductor and to satisfy the performance requirements. The delay buffer implemented by the three inverters shown in Fig. 3(a) determines the width of the transmitted signal.

Alternatively, the receiver circuit is a synchronous sense amplifier driven by a differential pair, as illustrated in Fig. 3(b) [23]. Due to the limited coupling between the two induc- tors, a synchronous sensing scheme is preferred. Synchronous receivers sample the received pulse within a specified time interval and therefore the effect of crosstalk noise is reduced and accidental switching due to glitches is prevented.

The differential pair (M3) provides the received signal to the synchronous sense amplifier. The signal received by the sense amplifier is sampled according to the $T_{\text {sample }}$ clock signal. The peak performance of the inductive link is determined by the duty cycle of the sampling clock. Alternatively, the sensitivity of the receiver is determined by the driving strength of the devices controlled by the clock signal $(M 2, M 6)$. The minimum device width that rectifies the signal and supports the desired data rate is utilised.

In the receiver, the sensed signal is biased and amplified through $V_{\text {bias }}$ and $R_{\text {bias }}$. For the $0.35 \mu \mathrm{m}$ receiver, a bias voltage of $V_{\text {bias }}=1.3 \mathrm{~V}$ ensures that the differential pair operates in the saturation region. An input resistance of $R_{\text {bias }}=1.5 \mathrm{k} \Omega$ is chosen for this circuit to produce a high input gain. Therefore, the width of the other devices in the circuit of the receiver is smaller decreasing power. Equivalently, for the $65 \mathrm{~nm}$ receiver the same approach is applied with a bias voltage of $V_{\text {bias }}=0.6 \mathrm{~V}$ and $R_{\text {bias }}=2 \mathrm{k} \Omega$.

\section{Simulation Results}

The operation of the heterogeneous links and power tradeoffs are demonstrated in this section. As power is the primary objective of the proposed inductive links, a power comparison with prior designs at $65 \mathrm{~nm}$ [15] and $0.35 \mu \mathrm{m}$ [23] technologies is performed. The key idea is to exploit the heterogeneity of the system to decrease power as compared to existing homogeneous links.

Both transceivers are simulated with Cadence Spectre [24], exhibiting a data rate of $1 \mathrm{Gbps}$. The transmitted data $T x_{\text {data }}$ and the received data $R x_{\text {data }}$ are illustrated in Figs. 4(a) and 4(b), respectively, for the uplink and downlink transceiver. A full swing signal at the nominal voltage of each tier is produced, without the usage of level shifters. For a specific physical distance $X$, coupling level $k$, and data rate, the power can be lowered by carefully sizing the devices in both the $T x$ and $R x$ circuits, considering the different sensitivity of these circuits when implemented at dissimilar technologies.

For the uplink receiver, the size of the devices $(M 2, M 3, M 6)$ that minimises the power yields a sensitivity threshold of $300 \mathrm{mV}$. Further lowering this sensitivity threshold, requires a higher input gain. A higher input gain can be achieved by increasing the device size of the amplification stage (M3) and the clock controlled devices $(M 2, M 6)$. This approach, nevertheless, results in larger devices and higher currents in the sensing tier, where the supply voltage is $3.3 \mathrm{~V}$. Thus, an alternative design methodology is followed. By increasing the size of $M 0$ and $M 1$ in the $65 \mathrm{~nm}$ transmitter to drive a higher current and using the minimum power sensitivity (i.e. $300 \mathrm{mV}$ ) for the $0.35 \mu \mathrm{m}$ receiver, the communication specifications are satisfied, without significantly increasing the power. 

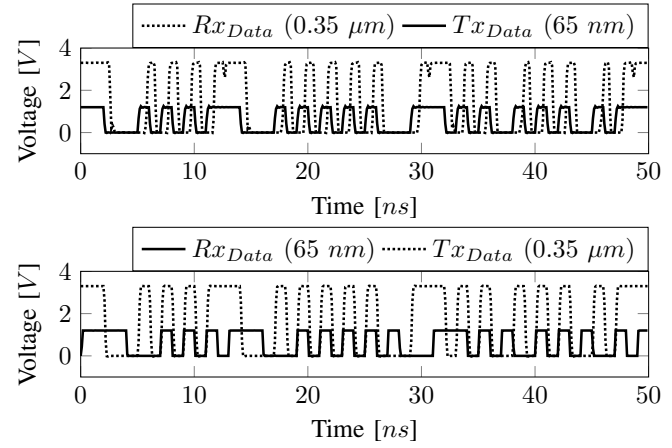

Fig. 4. Transient waveforms of the transmitted signal $T x_{\text {Data }}$ and the recovered signal $R x_{\text {Data }}$ for (a) the uplink transceiver and (b) the downlink transceiver.

Alternatively, the devices of the $65 \mathrm{~nm}$ receiver are not sized for minimum power but rather for highest sensitivity. Thus, the $65 \mathrm{~nm}$ receiver is designed to sense signals with amplitude as low as $75 \mathrm{mV}$. The resulting increase in the power of the receiver, however is compensated by the power savings due to the smaller size of the devices $M 0$ and $M 1$ in the $0.35 \mu \mathrm{m}$ transmitter. Consequently, as illustrated in the table of Fig. 3, the devices of the $65 \mathrm{~nm}$ tier are slightly larger than the devices of the $0.35 \mu \mathrm{m}$ tier, which are sized for minimum power. Therefore, the downlink transceiver benefits from the higher sensitivity of the $65 \mathrm{~nm}$ receiver which allows for a $70 \%$ decrease in the size of the devices of the $0.35 \mu \mathrm{m}$ transmitter.

The $65 \mathrm{~nm}$ transmitter consumes $P_{T x 65}=2.77 \mathrm{~mW}$ and the $0.35 \mu \mathrm{m}$ receiver $P_{R x 350}=2.16 \mathrm{~mW}$, tallying $P_{\text {uplink }}=$ $4.93 \mathrm{~mW}$ for the uplink circuit. Alternatively, for the downlink transceiver, $P_{T x 350}=8.18 \mathrm{~mW}$ are consumed by the $0.35 \mu \mathrm{m}$ transmitter and $P_{R x 65}=2.35 \mathrm{~mW}$ by the $65 \mathrm{~nm}$ receiver, respectively, for a total of $P_{\text {downlink }}=10.53 \mathrm{~mW}$.

Both $P_{\text {uplink }}$ and $P_{\text {downlink }}$ of the proposed transceivers exhibit a reduction of $89.1 \%$ and $73.9 \%$, respectively, compared to the transceiver in [23]. Considering the power within each tier, the power of the $65 \mathrm{~nm}$ tier is $P_{\text {tot }, 65}=5.12 \mathrm{~mW}$, as compared to $7.8 \mathrm{~mW}$ in [15], indicating a decrease of $34 \%$. Note, however, that a direct comparison with [15] is not feasible, since different transmitter and receiver circuits are used. Alternatively, the circuits implemented in the $0.35 \mu \mathrm{m}$ tier consume $P_{t o t, 350}=13.11 \mathrm{~mW}$. In [23], the power of the transmitter is $43 \mathrm{~mW}, 5.2 \times$ larger compared to $P_{T x 350}=$ $8.18 \mathrm{~mW}$. Concerning the receiver in [23], a reduction of $7.7 \%$ is observed for $P_{R x 350}$. These results demonstrate that by exploiting the sensitivity versus power tradeoff enabled by the use of heterogeneous technologies, a significant decrease in power is achieved for inter-tier communication with inductive links.

\section{CONClusion}

A heterogeneous transceiver for contactless links is presented for inter-tier communication between a processing and a sensing tier. Although heterogeneous technologies are considered, communication is achieved between the modules without the need of level shifters. Benefiting from technology heterogeneity, the power consumption of the transceivers is reduced to $4.93 \mathrm{~mW}$ for the uplink and to $10.53 \mathrm{~mW}$ for the downlink at $1 \mathrm{Gbps}$, compared to a $45.6 \mathrm{~mW}$ transceiver of a prototype $0.35 \mu \mathrm{m}$ system, while a decrease of $34 \%$ is observed compared to a $65 \mathrm{~nm}$ implementation.

\section{REFERENCES}

[1] D. Bandyopadhyay and J. Sen, "Internet of things: Applications and Challenges in Technology and Standardization," Wireless Personal Communications, Vol. 58, No. 1, pp. 49-69, April 2011.

[2] M. Wolf et al., "Technologies for 3D Wafer Level Heterogeneous Integration," Symposium on Design, Test, Integration and Packaging of MEMS/MOEMS, pp. 123-126, April 2008.

[3] V. F. Pavlidis and E. G. Friedman, Three-Dimensional Integrated Circuit Design, 1st ed. Morgan Kaufmann, 2009.

[4] H. Eltoukhy et al., "A 0.18- $\mu$ m CMOS Bioluminescence Detection Labon-Chip," IEEE Journal of Solid-State Circuits, Vol. 41, No. 3, pp. 651662, February 2006.

[5] M. Koyanagi et al., "High-Density Through Silicon Vias for 3-D LSIs," Proceedings of the IEEE, Vol. 97, No. 1, pp. 49-59, January 2009.

[6] J. Lau, "TSV Manufacturing Yield and Hidden Costs for 3D IC Integration," Proceedings of the IEEE Electronic Components and Technology Conference, pp. 1031-1042, June 2010.

[7] M.-T.-L. Aung et al., " $2.31-\mathrm{Gb} / \mathrm{s} / \mathrm{ch}$ Area-Efficient Crosstalk Canceled Hybrid Capacitive Coupling Interconnect for 3-D Integration,” IEEE Transactions On Very Large Scale Integration Systems, Vol. 24, No. 8, pp. 2703-2711, August 2016

[8] N. Miura et al., "A 195-Gb/s 1.2-W Inductive Inter-Chip Wireless Superconnect with Transmit Power Control Scheme for 3-D-Stacked System in a Package," IEEE Journal of Solid-State Circuits, Vol. 41, No. 1, pp. 23-34, December 2006.

[9] N. Miura and T. Kuroda, "A 1Tb/s 3W Inductive-Coupling Transceiver Chip," Proceedings of the IEEE/ACM Asia and South Pacific Design Automation Conference, pp. 92-93, January 2007.

[10] I. A. Papistas and V. F. Pavlidis, "Bandwidth-to-Area Comparison of Through Silicon Vias and Inductive Links for 3-D ICs," Proceedings of the IEEE European Conf. on Circuit Theory and Design, August 2015.

[11] W. R. Davis et al., "Demystifying 3D ICs: The Pros and Cons of Going Vertical," IEEE Design \& Test of Computers, Vol. 22, No. 6, pp. 498510 , November 2005

[12] A. Fazzi et al., "3-D Capacitive Interconnections with Mono-and BiDirectional Capabilities," IEEE Journal of Solid-State Circuits, Vol. 43 , No. 1, pp. 275-284, January 2008.

[13] N. Miura et al., "Inductive Coupled Communications," in Coupled Data Communication Techniques for High-Performance and Low-Power Computing, pp. 79-125, ISBN: 978-1-4419-6587-5. Springer, 2010.

[14] N. Miura et al., "A Scalable 3D Heterogeneous Multicore with an Inductive ThruChip Interface," IEEE Micro, Vol. 33, No. 6, pp. 6-15, December 2013.

[15] N. Miura et al., “A 1 TB/s 1 pJ/b 6.4 QDR Inductive-Coupling Interface Between 65-nm CMOS Logic and Emulated 100-nm DRAM," IEEE Journal on Emerging and Selected Topics in Circuits and Systems, Vol. 2, No. 2, pp. 249-256, May 2012.

[16] K. Niitsu et al., "An Inductive-Coupling Link for 3D Integration of a 90nm CMOS Processor and a 65nm CMOS SRAM," IEEE International Solid-State Circuits Conference, pp. 480-481, February 2009.

[17] D. Bol et al., "Green SoCs for a Sustainable Internet-of-Things," Proceedings of the IEEE Faible Tension Faible Consommation Conference, pp. 1-4, June 2013.

[18] United Microelectronics Corporation UMC. [Online]. Available: http://umc.com/

[19] ams AG. [Online]. Available: http://ams.com/

[20] C. Z. Goh et al., "A CMOS-Based ISFET Chemical Imager with AutoCalibration Capability," IEEE Sensors Journal, Vol. 11, No. 12, pp. 3253-3260, December 2011.

[21] Ansys Maxwell Online Help, v16, Ansys, Inc., December 2014.

[22] VeloceRF User Manual, v2, Helic, Inc., November 2013.

[23] N. Miura et al., "Analysis and Design of Inductive Coupling and Transceiver Circuit for Inductive Inter-Chip Wireless Superconnect," IEEE Journal of Solid-State Circuits, Vol. 40, No. 4, pp. 829-837, April 2005.

[24] Cadence Virtuoso Spectre Circuit Simulator Reference, v10.1.1, Cadence, Inc., June 2011. 